\title{
Incidence of positive peritoneal cytology in patients with endometrial carcinoma after hysteroscopy vs. dilatation and curettage
}

\author{
Andraz Dovnik¹, Bojana Crnobrnja¹, Branka Zegura1, Iztok Takac¹,2, Maja Pakiz¹ \\ ${ }^{1}$ University Clinic for Gynaecology and Perinatology, University Medical Centre Maribor, Maribor, Slovenia \\ ${ }^{2}$ Faculty of Medicine, University of Maribor, Maribor, Slovenia
}

Radiol Oncol 2017; 51(1): 88-93.

Received 30 January 2016

Accepted 28 April 2016

Correspondence to: Andraž Dovnik, M.D., University Clinic for Gynaecology and Perinatology, University Medical Centre Maribor, Ljubljanska 5, SI-2000 Maribor, Slovenia. Phone: +386 2321 2178; +386 31807 874; E-mail: andrazdovnik@gmail.com

Disclosure: No potential conflicts of interest were disclosed.

Background. The aim of the study was to compare the frequency of positive peritoneal washings in endometrial cancer patients after either hysteroscopy (HSC) or dilatation and curettage (D\&C).

Patients and methods. We performed a retrospective analysis of 227 patients who underwent either HSC (N=144) or $D \& C(N=83)$ and were diagnosed with endometrial carcinoma at the University Medical Centre Maribor between January 2008 and December 2014. The incidence of positive peritoneal cytology was evaluated in each group.

Results. There was no overall difference in the incidence of positive peritoneal washings after HSC or D\&C (HSC = $13.2 \% ; D \& C=12.0 \% ; p=0.803$ ). However, a detailed analysis of stage I disease revealed significantly higher rates of positive peritoneal washings in the HSC group ( $H S C=12.8 \% ; D \& C=3.4 \% ; p=0.046$ ). Among these patients, there was no difference between both groups considering histologic type (chi-square $=0.059 ; p=0.807$ ), tumour differentiation (chi-square $=3.709 ; p=0.156$ ), the time between diagnosis and operation ( $t=0.930 ; p=0.357$ ), and myometrial invasion (chi-square $=5.073 ; p=0.079$ ).

Conclusions. Although the diagnostic procedure did not influence the overall incidence of positive peritoneal washings, HSC was associated with a significantly higher rate of positive peritoneal cytology in stage I endometrial carcinoma compared to D\&C.

Key words: endometrial carcinoma; peritoneal cytology; FIGO staging; hysteroscopy; dilatation and curettage

\section{Introduction}

The diagnosis of endometrial cancer can be made preoperatively by obtaining a sample of endometrial tissue either with office endometrial biopsy, most commonly done with a Pipelle aspiration catheter, hysteroscopy (HSC), or dilatation and curettage $(\mathrm{D} \& \mathrm{C}){ }^{1}$ The latter two procedures are most commonly used in Slovenia. HSC has been shown to be highly accurate in diagnosing endometrial cancer ${ }^{2,3}$ and is considered a gold standard. ${ }^{1}$ Conflicting evidence has been published in the past regarding the risk of intraperitoneal spread of malignant cells after HSC with the use of distension media. ${ }^{47}$ In 2007 , a retrospective study from our institution reported a significantly higher incidence of positive peritoneal washings after HSC compared to D\&C. ${ }^{8}$ However, only 24 patients in this study had undergone HSC compared to 122 who were diagnosed with D\&C. ${ }^{8}$ In recent years, HSC has become an established diagnostic tool at our institution and is now performed more frequently than D\&C. The aim of our present study was to find out whether the difference in the incidence of positive peritoneal washings between HSC and D\&C persists after including a higher number of patients with hysteroscopy. 


\section{Patients and methods}

This retrospective study included all consecutive patients who had endometrial carcinoma diagnosed preoperatively with either D\&C or HSC between January 2008 and December 2014 at the University Medical Centre Maribor, Slovenia. The study included patients who had more than one D\&C or more than one HSC. Patients who had undergone both D\&C and HSC were excluded from the study. The study was approved by our institution's ethics committee (Approval No. 13-03/15, November 26, 2015). All patients signed a written informed consent that their medical records can be used for research matters retrospectively.

HSC was performed in the office setting or under general anesthesia. Saline solution warmed to body temperature was used as the distension medium. In the office setting, the distension medium was installed into the pressure cuff and the intrauterine pressure was set between $80-150 \mathrm{mmHg}$. Intrauterine pressure was controlled with the Vario Flow device (Pelta, Slovenia) in operative HSC. ${ }^{9}$ $D \& C$ was performed under general anesthesia. Curettage of the cervical canal and the uterine cavity was performed separately. Tissue samples for histologic examination were obtained during both procedures.

During the final surgery for endometrial carcinoma, samples of peritoneal washings from the pouch of Douglas were obtained for cytologic examination. Irrigation of the peritoneal cavity with saline solution was performed to obtain samples in cases with no free fluid. The samples were inspected by an expert cytopathologist. In cases of suspicious peritoneal cytology additional calretinin, MOC 31, HBME 1 and Ber-EP4 immunostaining was performed during the clarification process. In cases of small numbers of positive cells after immunostaining peritoneal cytology was described as suspicious. We therefore included suspicious results in the analysis of positive peritoneal cytology.

The primary statistical outcome was the incidence of positive peritoneal washings after HSC and after D\&C. A detailed analysis of tumour histopathologic characteristics was performed including histopathologic type, tumour differentiation, depth of myometrial invasion, lymphovascular invasion and FIGO stage. Different types of endometrial carcinomas were identified in the study population with endometrioid carcinoma representing the majority of cases $(\mathrm{N}=211 ; 93.0 \%)$. Other carcinomas (serous adenocarcinoma: $\mathrm{N}=8$;
TABLE 1. Histologic characteristics of the patients with endometrioid endometrial carcinoma ( $N=211$ ) and non-endometrioid endometrial carcinoma $(N=16)$

\begin{tabular}{|c|c|c|c|}
\hline Histologic parameter & $\begin{array}{l}\text { Endometrioid } \\
\text { endometrial } \\
\text { carcinoma }\end{array}$ & $\begin{array}{c}\text { Non-endometrioid } \\
\text { endometrial } \\
\text { carcinoma }\end{array}$ & $p^{*}$ \\
\hline Tumour differentiation & & & $<0.001$ \\
\hline G1 & $125(59.2 \%)$ & $2(13.3 \%)$ & \\
\hline G2 & $63(29.9 \%)$ & $4(26.6 \%)$ & \\
\hline G3 & $23(10.9 \%)$ & $9(60.0 \%)$ & \\
\hline \multicolumn{4}{|l|}{ Myometrial invasion } \\
\hline none & $8(3.8 \%)$ & $1(6.3 \%)$ & 0.888 \\
\hline less than $1 / 2$ & $123(58.3 \%)$ & 9 (56.2\%) & \\
\hline more than $1 / 2$ & 80 (37.9\%) & $6(37.5 \%)$ & \\
\hline \multicolumn{4}{|l|}{2009 FIGO stage } \\
\hline IA & $114(54.0 \%)$ & $7(43.8 \%)$ & 0.025 \\
\hline IB & $54(25.6 \%)$ & $1(6.3 \%)$ & \\
\hline$\|$ & $14(6.6 \%)$ & $2(12.5 \%)$ & \\
\hline IIIA & $7(3.3 \%)$ & $1(6.3 \%)$ & \\
\hline IIIB & $5(2.4 \%)$ & $0(0.0 \%)$ & \\
\hline$\| I C 1$ & $11(4.7 \%)$ & $2(12.5 \%)$ & \\
\hline IIIC2 & $0(0.0 \%)$ & $0(0.0 \%)$ & \\
\hline IVA & $1(0.5 \%)$ & $1(6.3 \%)$ & \\
\hline IVB & $5(2.4 \%)$ & $2(12.5 \%)$ & \\
\hline
\end{tabular}

*chi-square test

clear cell adenocarcinoma: $\mathrm{N}=8$ ) were assigned into non-endometriod group for the purpose of the study. Tumour differentiation was reported as good, moderate or poor. The depth of myometrial invasion was reported as no invasion, less than half of myometrium or more than half of myometrium. Patients treated before 2009 who had been staged according to the 1988 FIGO classification were restaged according to the new 2009 FIGO classification for statistical analysis. The time interval from diagnosis to final surgery was also analyzed.

Statistical analysis was performed with SPSS software version 22.0 (IBM, Armonk, NY, USA). Descriptive analysis, chi-square test and t-test of independent samples were performed as applicable. A $p$ value of less than 0.05 was considered statistically significant.

\section{Results}

Between January 2008 and December 2014, 266 patients had uterine cancer diagnosed with D\&C and/or HSC. 227 patients who had either HSC $(\mathrm{N}=$ 
TABLE 2. Histologic characteristics of the patients with endometrial carcinoma ( $N$ $=227$ ) who were diagnosed with hysteroscopy $(\mathrm{HSC})$ or dilatation and curettage (D\&C)

\begin{tabular}{|c|c|c|c|}
\hline Histologic parameter & $\operatorname{HSC}(N=144)$ & $D \& C(N=83)$ & $P$ value \\
\hline \multicolumn{4}{|l|}{ Histologic type } \\
\hline endometrioid adenocarcinoma & $137(95.1 \%)$ & $74(89.2 \%)$ & $0.090^{*}$ \\
\hline $\begin{array}{l}\text { non-endometrioid } \\
\text { adenocarcinoma }\end{array}$ & $7(4.9 \%)$ & $9(10.8 \%)$ & \\
\hline \multicolumn{4}{|l|}{ Tumour differentiation } \\
\hline G1 & 90 (62.5\%) & $37(45.1 \%)$ & $0.012^{*}$ \\
\hline G2 & $40(27.8 \%)$ & $27(32.9 \%)$ & \\
\hline G3 & $14(9.7 \%)$ & $18(22.0 \%)$ & \\
\hline \multicolumn{4}{|l|}{ Myometrial invasion } \\
\hline none & $5(3.5 \%)$ & $4(4.8 \%)$ & $0.726^{*}$ \\
\hline less than $1 / 2$ & $82(56.9 \%)$ & $50(60.2 \%)$ & \\
\hline more than $1 / 2$ & $57(39.6 \%)$ & 29 (34.9\%) & \\
\hline \multicolumn{4}{|l|}{ Lymphovascular invasion } \\
\hline present & $20(13.9 \%)$ & $14(16.9 \%)$ & $0.545^{*}$ \\
\hline absent & $124(86.1 \%)$ & $69(83.1 \%)$ & \\
\hline $\begin{array}{l}2009 \text { FIGO stage } \\
\text { IA } \\
\text { IB } \\
\text { II } \\
\text { IIIA } \\
\text { IIIB } \\
\text { IIIC } 1 \\
\text { IIIC } 2 \\
\text { IVA } \\
\text { IVB }\end{array}$ & $\begin{array}{c}75(52.1 \%) \\
42(29.2 \%) \\
10(6.9 \%) \\
1(0.7 \%) \\
3(2.1 \%) \\
9(6.3 \%) \\
0(0.0 \%) \\
1(0.7 \%) \\
3(2.1 \%)\end{array}$ & $\begin{array}{c}46(55.4 \%) \\
13(15.7 \%) \\
6(7.2 \%) \\
7(8.4 \%) \\
2(2.4 \%) \\
4(4.8 \%) \\
0(0.0 \%) \\
1(1.2 \%) \\
4(4.8 \%)\end{array}$ & $0.040^{*}$ \\
\hline $\begin{array}{l}\text { Peritoneal cytology } \\
\text { positive or suspicious } \\
\text { negative }\end{array}$ & $\begin{array}{c}19(13.2 \%) \\
125(86.8 \%)\end{array}$ & $\begin{array}{l}10(12.0 \%) \\
73(88.0 \%)\end{array}$ & $0.803^{*}$ \\
\hline $\begin{array}{l}\text { Time from diagnosis to } \\
\text { operation (days) }\end{array}$ & $35.8 \pm 13.8$ & $32.8 \pm 15.4$ & $0.140^{* *}$ \\
\hline
\end{tabular}

*chi-square test

** independent sample t-test ing the diagnostic evaluation $(13.2 \%$ after $\mathrm{HSC}$, $12.0 \%$ after $\mathrm{D} \& \mathrm{C}$; chi-square $=0.062 ; \mathrm{p}=0.803)$.

The groups (HSC vs. D\&C) did not differ in the prevalence of histologic types of the tumour, depth of myometrial invasion and lymphovascular invasion (Table 2). However, there were significant differences in tumour differentiation as well as FIGO stage, with more patients having FIGO stage I disease in the hysteroscopy group (Table 2). Due to this difference we conducted analysis only in the subgroup of patients with stage I disease. The HSC and $D \& C$ groups of stage I patients did not differ in tumour differentiation, the prevalence of histologic types of the tumour, the time from diagnosis to operation and in myometrial invasion. A separate evaluation of patients with stage I tumours showed that $12.8 \%$ in the HSC group and only $3.4 \%$ in the D\&C group had positive peritoneal washings. This difference was statistically significant (chi-square $=$ 2.422; $\mathrm{p}=0.046)$ (Table 3).

One out of 15 FIGO stage I patients with positive peritoneal washings after hysteroscopy had disease recurrence by the end of April 2015 (mean follow-up 40.2 months). Neither of the two FIGO stage I patients with positive peritoneal washings after D\&C had disease recurrence in the same period (mean follow-up 39.5 months).

\section{Discussion}

The possibility of microscopic intraperitoneal spread of endometrial cancer cells after hysteroscopy has been a subject of debate for more than a decade. In our study, we did not find an increased incidence of positive peritoneal washings after hysteroscopy in comparison to D\&C in the overall study population of patients with endometrial carcinoma. Several other studies similarly found no association between hysteroscopy and an increased rate of positive peritoneal cytology. ${ }^{5,6} \mathrm{On}$ the other hand, Bradley et al. ${ }^{7}$ reported a higher frequency of positive or suspicious peritoneal cytology after hysteroscopy compared to blind endometrial sampling using logistic regression controlling for confounders of grade and stage. They also reported a higher rate of disease upstaging (according to the 1988 FIGO staging system) after hysteroscopy attributed solely to the positive cytology. Similar results have been reported by Zerbe $e t$ $a l .{ }^{10}$ and Obermair et al. ${ }^{11}$ In a study conducted at our institution in $2007^{8}$, positive peritoneal cytology was present in $12.5 \%$ of patients after hysteroscopy and only in $1.6 \%$ after D\&C. The difference 
was statistically significant (chi-square $=4.2455$; $\mathrm{p}<0.005)$.

In a meta-analysis of nine trials including 1015 patients with confirmed endometrial carcinoma, Polyzos et al. ${ }^{12}$ evaluated the rate of positive peritoneal washings after hysteroscopy in comparison to other diagnostic procedures or no diagnostic procedures. They concluded that the frequency of positive peritoneal washings was significantly higher after hysteroscopy. The analysis also revealed a higher rate of disease upstaging based only on the positive peritoneal cytology. A detailed literature search performed by Guralp and Kushner ${ }^{13}$ revealed $0-83 \%$ of positive peritoneal cytology after hysteroscopy and 0-13.6\% after D\&C. However, the authors emphasized a number of unanswered questions regarding the type and volume of distension medium, intrauterine pressure during the procedure, time interval between hysteroscopy and definitive surgery, stage, grade of the disease and duration of the procedure. ${ }^{13}$ Another metaanalysis by Chang et al. ${ }^{14}$ also reported on higher rates of positive peritoneal cytology after hysteroscopy. Nevertheless, a detailed analysis of patients with stages I or II failed to show significantly higher rates of positive peritoneal cytology in patients who had hysteroscopy.

Interestingly, our results showed a significantly higher incidence of positive or suspicious peritoneal cytology in patients with stage I disease who were diagnosed with hysteroscopy compared to those diagnosed with D\&C. This is an unexpected finding because the disease at this stage is confined to the uterus. For example, only $3.3 \%$ of patients with stage I and II endometrial cancer in a large retrospective analysis by Garg et al. ${ }^{15}$ had positive peritoneal cytology. In our study, the rate of positive or suspicious peritoneal cytology in stage I disease was $3.3 \%$ in the D\&C group but as much as $12.1 \%$ in the hysteroscopy group. Positive or suspicious peritoneal cytology was shown to be more frequent after hysteroscopy in endometrial carcinoma patients who would be staged as FIGO IA in the new staging system by Obermair et al. ${ }^{11}$ On the other hand, Biewenga et al. ${ }^{6}$ showed no association between hysteroscopy and the rate of positive peritoneal washings in stage I disease.

Saline solution was used as the distension medium in all our patients in the hysteroscopy group. Hysteroscopy with saline solution was specifically linked to a higher rate of positive peritoneal cytology in a meta-analysis by Polyzos et al. ${ }^{12}$ In a metaanalysis by Chang et al. ${ }^{14}$, the distension medium was either saline solution or $5 \%$ glucose solution.
TABLE 3. Histologic characteristics of the patients with stage 1 endometrial carcinoma ( $\mathrm{N}=187$ ) who were diagnosed with hysteroscopy $(\mathrm{HSC})$ or dilatation and curettage (D\&C)

\begin{tabular}{|c|c|c|c|}
\hline Stage 1 & HSC (N = 117) & $D \& C(N=59)$ & $P$ value \\
\hline \multicolumn{4}{|l|}{ Histologic type } \\
\hline endometrioid adenocarcinoma & $112(95.7 \%)$ & $56(94.9 \%)$ & \multirow[t]{2}{*}{$0.807^{*}$} \\
\hline $\begin{array}{l}\text { non-endometrioid } \\
\text { adenocarcinoma }\end{array}$ & $5(4.3 \%)$ & $3(5.1 \%)$ & \\
\hline \multicolumn{4}{|l|}{ Tumour differentiation } \\
\hline G1 & $75(64.1 \%)$ & $29(49.2 \%)$ & \multirow[t]{3}{*}{$0.156^{*}$} \\
\hline G2 & $32(27.4 \%)$ & $22(37.3 \%)$ & \\
\hline G3 & $10(8.5 \%)$ & $8(13.5 \%)$ & \\
\hline \multicolumn{4}{|l|}{ Myometrial invasion } \\
\hline none & $5(4.3 \%)$ & $3(5.1 \%)$ & \multirow[t]{3}{*}{$0.079 *$} \\
\hline less than $1 / 2$ & $71(60.7 \%)$ & $45(76.2 \%)$ & \\
\hline more than $1 / 2$ & $41(35.0 \%)$ & $11(18.6 \%)$ & \\
\hline $\begin{array}{l}\text { Peritoneal cytology } \\
\text { positive or suspicious } \\
\text { negative }\end{array}$ & $\begin{array}{l}15(12.8 \%) \\
102(87.2 \%)\end{array}$ & $\begin{array}{l}2(3.4 \%) \\
57(96.6 \%)\end{array}$ & $0.046 *$ \\
\hline $\begin{array}{l}\text { Time from diagnosis to } \\
\text { operation (days) }\end{array}$ & $35.6 \pm 14.0$ & $33.1 \pm 12.7$ & $0.255^{* *}$ \\
\hline
\end{tabular}

*chi-square test

** independent sample t-test

Neither of these two meta-analyses found a connection between intrauterine pressure during hysteroscopy higher than $100 \mathrm{mmHg}$ and a higher incidence of positive peritoneal cytology. ${ }^{12,14}$ In our study, the exact intrauterine pressure during hysteroscopy was not known for each patient individually due to the retrospective nature of the analysis.

The time interval between the diagnostic procedure and definitive surgery was similar in patients with positive and negative peritoneal cytology in our study. This is in line with evidence from another retrospective study in 196 patients with endometrial cancer diagnosed with hysteroscopy. ${ }^{16}$

Based on the data from our retrospective study, we cannot give a definite reason for the significantly higher incidence of positive peritoneal washings after HSC compared to D\&C in stage I disease. We used saline solution as the distension medium, which has been previously associated with higher rates of positive peritoneal cytology. ${ }^{12}$ Unfortunately, we do not have the exact information on the intrauterine pressure during HSC and the duration of the diagnostic procedure for each patient and therefore we cannot draw conclusions about the influence of these factors on peritoneal cytology.

Another important limitation of our study is the inclusion of suspicious peritoneal cytology in the 
positive peritoneal cytology group. Even after immunostaining most of the cases without evident malignant cells remained cytologically suspicious because positive immune reaction was seen in only a small fraction of cells. However, it is not easy to differentiate positive from suspicious cytology because severe atypia of reactive mesothelial cells may be interpreted as suspicious. We are aware of this methodological limitation and should aim to lower the incidence of suspicious peritoneal cytology in the future firstly by obtaining sufficient amount of fluid for cytological analysis during final surgery and secondly with accurate cytological diagnosis. Some published research on this subject also included positive and suspicious cytology in the same group. ${ }^{78,11}$

Our data show that among patients with positive or suspicious peritoneal washings after hysteroscopy, in FIGO stage I patients, one out of 15 had local disease recurrence during follow-up of approximately 40 months, whereas neither of the two with positive washings after $\mathrm{D} \& \mathrm{C}$ had the recurrence. As these numbers are small, further research is necessary to draw relevant conclusions. Conflicting results exist in the literature regarding the prognostic significance of positive peritoneal cytology. ${ }^{17,18}$ The updated FIGO staging system from 2009 excluded positive peritoneal cytology as a stage defining variable. Previously, all patients with positive peritoneal cytology were upstaged to stage IIIA. ${ }^{19}$ In an analysis of 14,704 patients, Garg et al. ${ }^{15}$ reported peritoneal cytology to be associated with survival in univariate analysis along with race, age, histology, grade and the number of removed lymph nodes. In multivariate analysis, positive peritoneal cytology remained an independent prognostic factor in stages I and II. Shiozaki et al..$^{20}$ studied the influence of positive peritoneal washings on the prognosis of 265 patients with stage I endometrial cancer. Progression-free survival was significantly lower in the group with positive peritoneal cytology. Other factors associated with progression-free survival in univariate analysis were lymph node dissection and vessel permeation, but positive peritoneal cytology was the most influential factor. ${ }^{20}$ Disease-free survival has been shown to be $91 \%$ in FIGO stage I patients and $52.5 \%$ in those with FIGO stage II, III and IV. ${ }^{21}$ In the study by Garg et al., survival in patients with stage I endometrioid adenocarcinoma with positive peritoneal washings was significantly poorer than in patients with negative peritoneal washings $(88.2 \%$ vs. $98.6 \%) .{ }^{15}$

In conclusion, the diagnostic procedure did not influence the overall incidence of positive peritone- al washings in our study. However, hysteroscopy was associated with a significantly higher rate of positive peritoneal cytology in stage I endometrial carcinoma. Although statistically significant, this finding must be interpreted with caution because of the small sample size of this subgroup. In addition, it is still not known whether iatrogenic dissemination of malignant cells bears the same influence on disease prognosis as spontaneous dissemination. Despite being excluded as the stage defining variable, peritoneal cytology should still be reported separately as requested by FIGO. ${ }^{15}$ We believe that additional trials are needed to further clarify the prognostic value of positive peritoneal cytology after hysteroscopy, particularly in the early stages of endometrial cancer.

\section{References}

1. Burke WM, Orr J, Leitao M, Salom E, Gehrig P, Olawaiye AB, et al. Society of Gynecologic Oncology Clinical Practice Committee. Endometrial cancer: a review and current management strategies: part I. Gynecol Oncol 2014; 134: 385-92.

2. Clark TJ, Voit D, Gupta JK, Hyde C, Song F, Khan KS. Accuracy of hysteroscopy in the diagnosis of endometrial cancer and hyperplasia: a systematic quantitative review. JAMA 2002; 288: 1610-21.

3. Gkrozou F, Dimakopoulos G, Vrekoussis T, Lavasidis L, Koutlas A, Navrazoglou I, et al. Hysteroscopy in women with abnormal uterine bleeding: a metaanalysis on four major endometrial pathologies. Arch Gynecol Obstet 2015; 291: 1347-54.

4. Gu M, Shi W, Huang J, Barakat RR, Thaler HT, Saigo PE. Association between initial diagnostic procedure and hysteroscopy and abnormal peritoneal washings in patients with endometrial carcinoma. Cancer 2000; 90: 143-7.

5. Selvaggi L, Cormio G, Ceci O, Loverro G, Cazzolla A, Bettocchi S. Hysteroscopy does not increase the risk of microscopic extrauterine spread in endometrial carcinoma. Int J Gynecol Cancer 2003; 13: 223-7.

6. Biewenga $\mathrm{P}$, de Blok S, Birnie E. Does diagnostic hysteroscopy in patients with stage I endometrial carcinoma cause positive peritoneal washings? Gynecol Oncol 2004; 93: 194-8.

7. Bradley WH, Boente MP, Brooker D, Argenta PA, Downs LS, Judson PL, et al. Hysteroscopy and cytology in endometrial cancer. Obstet Gynecol 2004; 104: 1030-3.

8. Takac I, Zegura B. Office hysteroscopy and the risk of microscopic extrauterine spread in endometrial cancer. Gynecol Oncol 2007; 107: 94-8.

9. Tomaževič T. Operative hysteroscopy. In: Ribič-Pucelj M, editor. Endoscopic surgery in gynaecology. Radovljica: Didakta; 2007. p. 251-7.

10. Zerbe MJ, Zhang J, Bristow RE, Grumbine FC, Abularach S, Montz FJ Retrograde seeding of malignant cells during hysteroscopy in presumed early endometrial cancer. Gynecol Oncol 2000; 79: 55-8.

11. Obermair A, Geramou M, Gucer F, Denison U, Graf AH, Kapshammer E, et al. Does hysteroscopy facilitate tumor cell dissemination? Incidence of peritoneal cytology from patients with early stage endometrial carcinoma following dilatation and curettage (D \& C) versus hysteroscopy and D \& C. Cancer 2000; 88: 139-43.

12. Polyzos NP, Mauri D, Tsioras S, Messini Cl, Valachis A, Messinis IE. Intraperitoneal dissemination of endometrial cancer cells after hysteroscopy: a systematic review and meta-analysis. Int J Gynecol Cancer 2010; 20: 261-7.

13. Guralp O, Kushner DM. latrogenic transtubal spill of endometrial cancer: risk or myth. Arch Gynecol Obstet 2011; 284: 1209-21. 
14. Chang YN, Zhang Y, Wang YJ, Wang LP, Duan H. Effect of hysteroscopy on the peritoneal dissemination of endometrial cancer cells: a meta-analysis. Fertil Steril 2011; 96: 957-61.

15. Garg G, Gao F, Wright JD, Hagemann AR, Mutch DG, Powell MA. Positive peritoneal cytology is an independent risk-factor in early stage endometrial cancer. Gynecol Oncol 2013; 128: 77-82.

16. Juhasz-Böss I, Fehm T, Nauth A, Becker S, Rothmund R, Gardanis K, et al. Number of hysteroscopies and the time interval between hysteroscopy and surgery: influence on peritoneal cytology in patients with endometrial cancer. Anticancer Res 2010; 30: 2425-30.

17. Saga Y, Imai M, Jobo T, Kuramoto H, Takahashi K, Konno R, et al. Is peritoneal cytology a prognostic factor of endometrial cancer confined to the uterus? Gynecol Oncol 2006; 103: 277-80.

18. Fadare O, Mariappan MR, Hileeto D, Wang S, McAlpine JN, Rimm DL. Upstaging based solely on positive peritoneal washing does not affect outcome in endometrial cancer. Mod Pathol 2005; 18: 673-80.

19. Pecorelli S. Revised FIGO staging for carcinoma of the vulva, cervix, and endometrium. Int J Gynecol Obstet 2009; 105: 103-4.

20. Shiozaki T, Tabata T, Yamada T, Yamamoto Y, Yamawaki T, Ikeda T. Does positive peritoneal cytology not affect the prognosis for stage I uterine endometrial cancer? The remaining controversy and review of the literature. Int J Gynecol Cancer 2014; 24: 549-55.

21. Tejerizo-García A, Jiménez-López JS, Muñoz-González JL, Bartolomé-Sotillos S, Marqueta-Marqués L, López-González G, et al. Overall survival and disease-free survival in endometrial cancer: prognostic factors in 276 patients. Onco Targets Ther 2013; 9: 1305-13. 